\title{
Microfluidic Blood Typing Chip with Voltammetry Measurement
}

\author{
Yaw-Jen Chang , Xin-Miao Zhou, Hsiu-Rong Yen and Chih-Ling Yeh \\ Department of Mechanical Engineering, Chung Yuan Christian University, Chung Li District, Taoyuan City, Taiwan
}

\begin{abstract}
Blood typing is a critical test in many medical procedures, especially for blood transfusion. Incompatible red blood cells (RBCs) in transfusion might result in intravascular hemolysis and even cause recipients death. The test result of blood typing can be determined by observing the agglutination of RBCs. In this study, a microfluidic blood typing chip with interdigitated electrodes is presented. $\mathrm{ZnO}$ nanowires were synthesized on the interdigitated electrodes so that voltammetry measurement was employed to determine the test results of blood typing. The experimental results show that the discrimination between agglutination and non-agglutination was obvious and successful determinations of RBC agglutinations in blood typing tests were achieved by means of voltammetry measurement.
\end{abstract}

\section{Introduction}

Blood is a body fluid in humans for delivering nutrients and oxygen to the cells and, simultaneously, transporting metabolic waste products away. In case of heavy bleeding, blood transfusion is a medical treatment to replace the lost blood components. Before the transfusion, blood typing is a critical test to ensure the serological compatibility of a donor and a recipient [1]. Incompatible red blood cells (RBCs) in transfusion, i.e. mistyping of the ABO blood group, might result in intravascular hemolysis and even cause recipient's death [2]. Blood typing is based on the antigen-antibody interaction. If antigens on the surface of RBCs correspond to antibodies in the serum, agglutination of RBCs occurs. Therefore, the test result of blood typing can be determined by observing the agglutination of RBCs with the naked eyes.

Currently, many traditional blood typing methods are available $[3,4]$. Fully automated blood typing systems are used in blood banks for routine tests. However, manual operation using 96-well microtiter plate is still the main approach in hospital transfusion services or laboratories. Besides, many researchers proposed different approaches for blood typing. Kline et al. [1] utilized the microfluidic droplet technique to encapsulate RBCs, antibody, and buffer in droplets to perform multiple agglutination assays. Makulska et al. [5] presented a method to determine the agglutination by measuring the speed of flow of microdroplets, containing RBCs and antibodies, in the microchannel.

Microfluidic systems have many advantages, including smaller consumption volume of reagents, shorter reaction time, and parallel operation in a single chip. Recently, microfluidic techniques attract a lot of interest and find important applications in chemistry and biology. Microfluidic chips for blood typing allow the operation sequence to be conducted automatically through the manipulation of microfluids. Nevertheless, a robust determination of the extent of RBC agglutination, instead of visual judgment, remains to be solved.

Over the past few years, interdigitated electrode (IDE) is a widely used structure as sensing component. Alternatively, nanowires (NWs) are also thought of as ideal elements for sensing devices and have been widely employed in a wide variety of fields, such as gas sensing $[6,7]$, chemical sensing [8], and biomolecule sensing $[9,10]$. Among the nanowire materials, zinc oxide $(\mathrm{ZnO})$ is a chemically and thermally stable n-type semiconductor material. Besides, this semiconductor is environmentally friendly and has several favorable properties, including high electron mobility, high melting point, good transparency, and large piezoelectric response. Many formation methods of $\mathrm{ZnO}$ nanowires have been developed [11]. Hydrothermal synthesis is a simple and popular method [12].

In this study, a microfluidic blood typing chip was designed for measuring RBC agglutination with voltammetry technique. Moreover, the voltammetry measurement was carried out using an interdigitated electrode on which $\mathrm{ZnO}$ nanowires were synthesized by hydrothermal method.

\section{Materials and methods}

\subsection{Chip design}

Blood typing is based on the antigen-antibody interaction. Agglutination is then observed to determine the serological compatibility of bloods. In this study, biomaterials, including A cell, B cell, anti-A, and anti-B, 
were purchased from Formosa Biomedical Technology Corp. (Taiwan) for carrying out the bioassays.

This microfludic blood typing chip, as shown in Figure 1, is comprised of five injection inlets for loading the necessary reagents (blood cell and antibody) and one detection chamber where the voltammetry measurement is performed. The injected reagents traverse the main channel to the detection chamber. On the way, the reagents pass through the special shaped mixing channels allowing both reagents to be mixed thoroughly and rapidly. Inside the detection chamber, there exists an interdigitated electrode array acting as sensor for voltammetry measurement of blood agglutination. The IDE array was characterized by 4 interdigitated fingers on each side of device with finger width of $250 \mu \mathrm{m}$, length of $3.9 \mathrm{~mm}$, and gap of $250 \mu \mathrm{m}$. On the surface of IDE array, $\mathrm{ZnO}$ nanowires were synthesized to promote the sensitivity of measurement. This nanowires-synthesized IDE array is denoted as NW-IDE. The voltammetry measurement was performed using 4145B Semiconductor Parameter Analyzer.

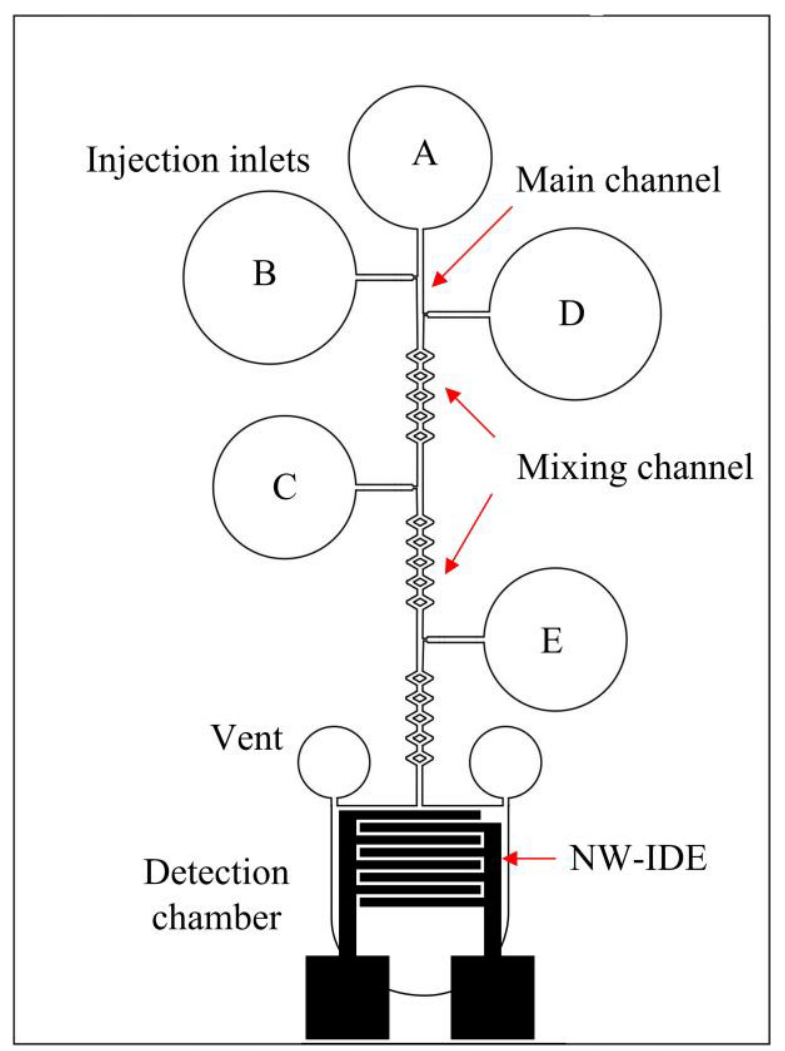

Figure 1. Design of microfluidic blood typing chip.

The width of the main channel is $200 \mu \mathrm{m}$. All the microfluidic components such as channel and chamber have depth of $200 \mu \mathrm{m}$. The reagents were driven using a syringe pump. Figure 2 shows the experimental setup.

\subsection{Chip fabrication}

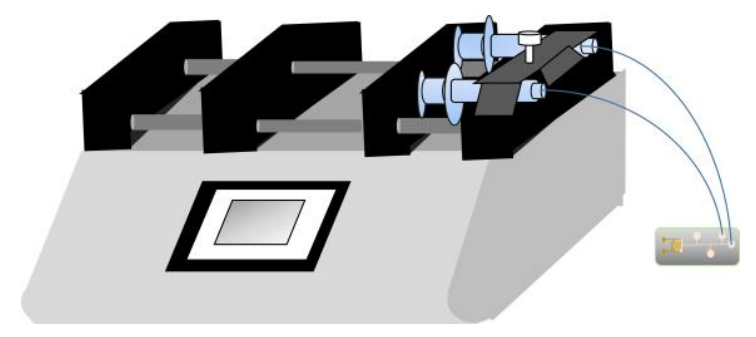

Figure 2. Experimental setup.

This proposed chip includes a microchannel layer allowing microfluids to flow and a substrate with NWIDE. This chip was produced by bonding the microchannel layer on the substrate.

\subsubsection{Fabrication of microchannel layer}

To fabricate the microchannel layer, the technique of standard SU-8 molding was used, as shown in Figure 3. A SU-8 mold with the opposite pattern of all microfluidic components was prepared first and, thus, the microfluidic layer was formed by casting polydimethylsiloxane (PDMS) in the mold. Then, the PDMS microchannel layer was left to cure at $95^{\circ} \mathrm{C}$ in an oven for $1 \mathrm{~h}$. Finally, it was carefully peeled from the SU-8 mold. This layer is denoted as PDMS1.

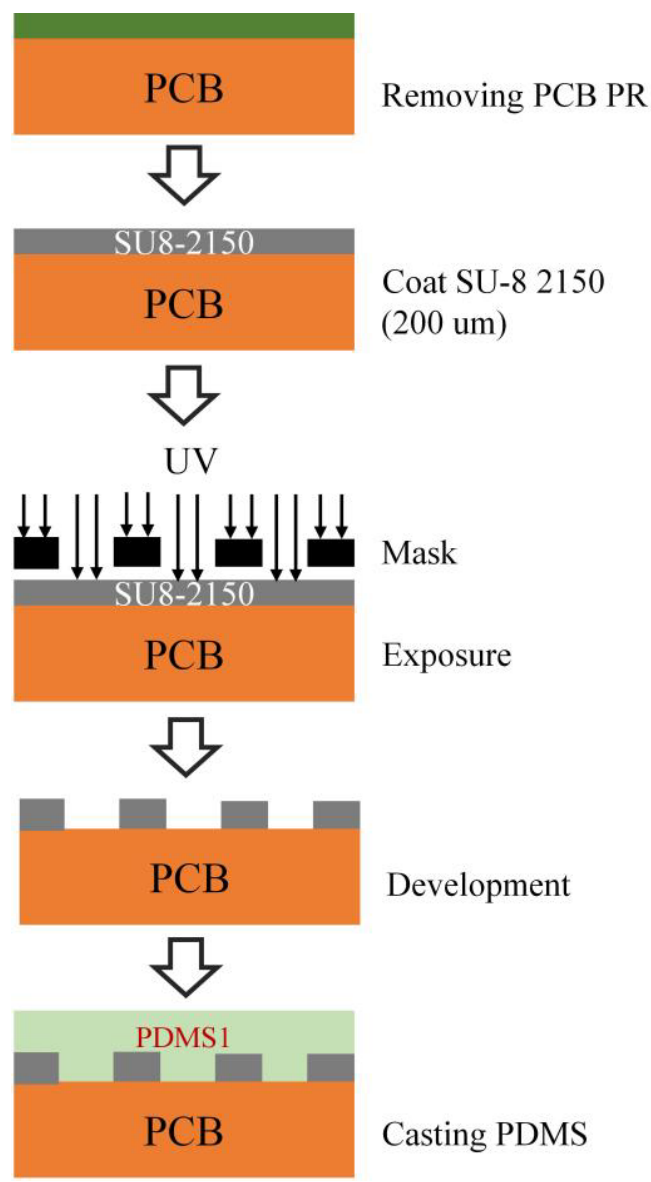

Figure 3. Fabrication process of microchannel layer. 


\subsubsection{Fabrication of NW-IDE}

Figure 4 shows the fabrication steps of NW-IDE. Printed circuit board (PCB) has a conducting layer typically made of thin copper foil. Hence, PCB was used as the substrate and its $\mathrm{Cu}$ film was etched to form the interdigitated electrodes. The designed pattern of IDE array was first transferred onto the photoresist layer of PCB by photolithography process. Then, etching the copper-conducting layer of PCB through the conventional PCB treatment generated the IDE structure.

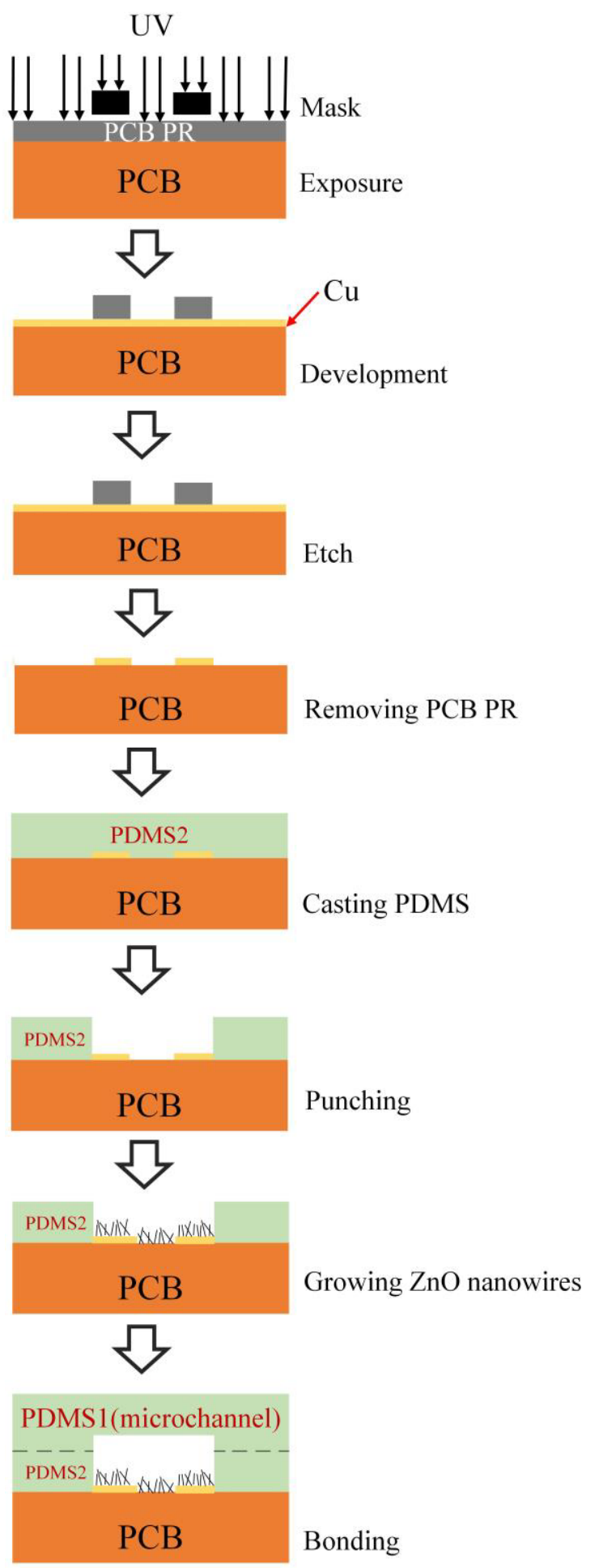

Figure 4. Fabrication process of NW-IDE.
The next step is the crystal growth of $\mathrm{ZnO}$ nanowires on the entire area of IDE array. However, $\mathrm{ZnO}$ must be constrained to grow only at the region of detection chamber. Otherwise, nanowires would influence the bonding between the microchannel layer and the substrate. Thus, prior to the growth of $\mathrm{ZnO}$ nanowires, a thin layer of PDMS was spun on the PCB substrate. This layer is denoted as PDMS2. After PDMS2 was cured in an oven, we opened a round hole in the PDMS2 using a hole punch, which is a kind of craft tool, to make the IDE array exposed for the growth of $\mathrm{ZnO}$ nanowires.

The conventional hydrothermal method was adopted. First, $\mathrm{ZnO}$ seed solution was prepared by mixing $0.328 \mathrm{~g}$ of zinc acetate dehydrate (J.T.Baker ${ }^{\circledR}$, purchased from Avantor Performance Materials, USA) with $150 \mathrm{~mL}$ of methanol, and stirring and heating. After the temperature of mixture raised to $60^{\circ} \mathrm{C}, 0.12 \mathrm{~g}$ of $\mathrm{NaOH}$ and $100 \mathrm{~mL}$ of methanol were added slowly. Heating was stopped when the solution became white muddy mixture. However, stirring was continued until the solution cooled down to the room temperature. Thereupon $\mathrm{ZnO}$ seed solution was spread on the surface of IDE array, followed by fifteen-minute dehydration baking in a $130^{\circ} \mathrm{C}$ oven. After the chip surface was dried, careful check on whether the electrode surface is entirely covered by $\mathrm{ZnO}$ seed film was made using microscope.

$\mathrm{ZnO}$ growing solution was formulated with $0.46 \mathrm{~g}$ of $\mathrm{Zn}\left(\mathrm{NO}_{3}\right)_{2}$ (analytical reagent grade, $98 \%$, Acros Organics, USA) and $0.2 \mathrm{~g}$ of $\mathrm{C}_{6} \mathrm{H}_{12} \mathrm{~N}_{4}$ (Showa Chemical Industry Co., Ltd., Japan) dissolved in $80 \mathrm{~mL}$ of DI water. Then, the $\mathrm{ZnO}$ seeded substrate was fixed facing down and placed in the growing solution. The lid of the vessel was sealed to prevent evaporation. Reaction was carried out at $95^{\circ} \mathrm{C}$ for $4 \mathrm{~h}$. An additional 4-h reaction was conducted after the solution was changed. Finally, the substrate was taken out from the vessel and rinsed with DI water, followed by drying with nitrogen.

Finally, the microchannel layer (PDMS1) was bonded on the PDMS layer of substrate (PDMS2) using oxygen plasma treatment.

\subsection{Bioassay}

Four sets of blood typing bioassays were conducted: (1) A cell and anti-A; (2) A cell and anti-B; (3) B cell and anti-A; (4) B cell and anti-B. For each experimental design, three chips were prepared for the purpose of repetitious experiments. The voltammetry measurement was then performed.

In this study, only two injection inlets were used, i.e., inlet $\mathrm{A}$ and inlet $\mathrm{D}$. The other three inlets can be utilized for the introduction of other reagents. For example, the manual polybrene (MP) technique for blood typing, which is widely adopted in Asia, requires other reagents such as low ionic medium (LIM), polybrene, and resuspension solution. The LIM can promote the reaction between RBC antigens and antibodies if they are associated. The function of polybrene is to cause nonspecific RBC aggregation. However, the resuspension solution is for reversing the non-specific polybreneinduced aggregation. These reagents must be added in a 
specific order. The design of this proposed chip is ready for conducting the MP test.

\section{Results and discussion}

If type A RBCs expose to anti-A, agglutination must occur. Similar result must be observed for the experiment of type B RBCs and anti-B. However, for nonagglutination situations, such as type A exposing to anti$\mathrm{B}$, most of the reactant solution should not display evident agglutinated blood cells.

\subsection{Photo image}

Photo image is a common evidence of blood typing result. Figures 5-1 to 5-4 show the photo images of our bioassays. Part (a) in each figure is the image of entire chip; while part (b) and (c) are the detailed images of detection chamber. In addition, front light source was adopted for taking photo of part (b). On the contrary, back light source was adopted for part (c).

As shown in Figures 5-1 and 5-4, red spots on NWIDE can be observed. They are the RBC agglutinations. However, it is difficult to determine the blood typing result with naked eyes. Since small amount of blood (3.6 $\mu \mathrm{L}$ ) was used in each bioassay, agglutination became unobvious.
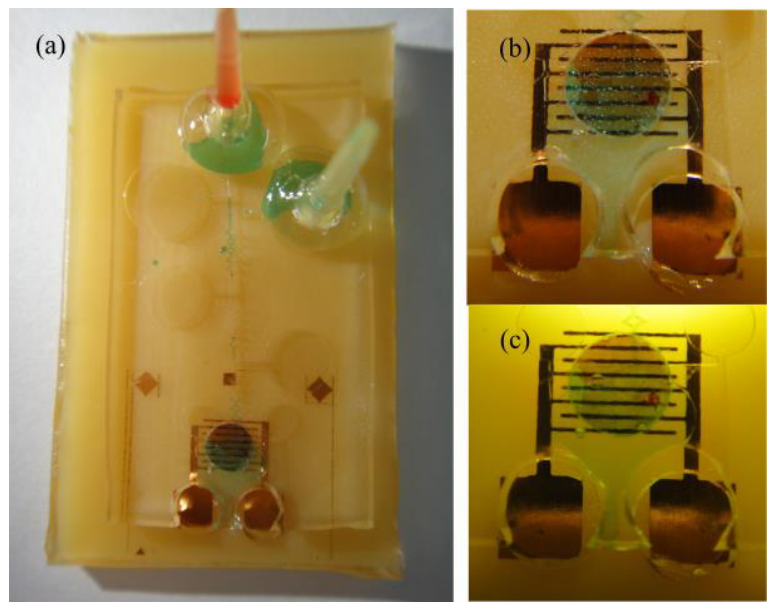

Figure 5-1. Agglutination (A cell and anti-A).
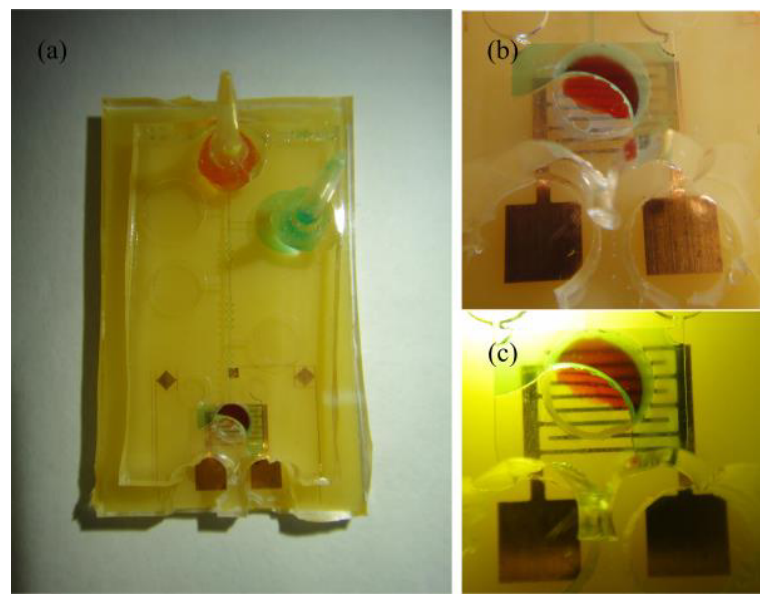

Figure 5-2. Non-agglutination (B cell and anti-A).
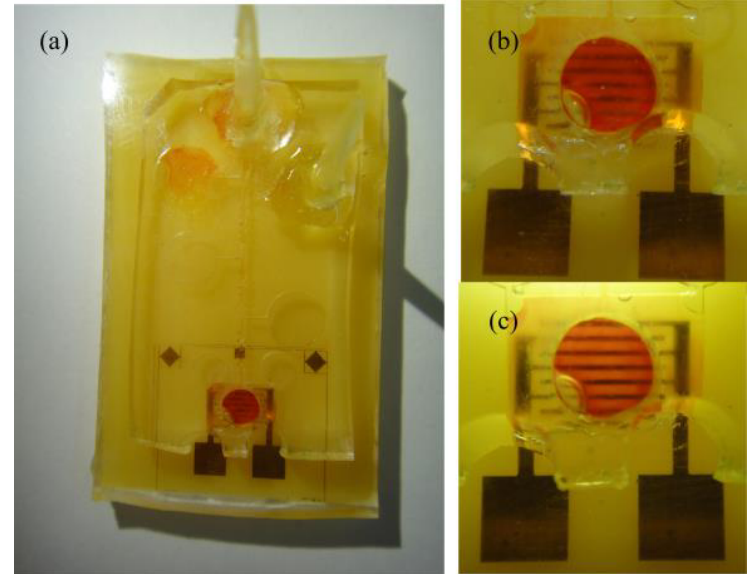

Figure 5-3. Non-agglutination (A cell and anti-B).
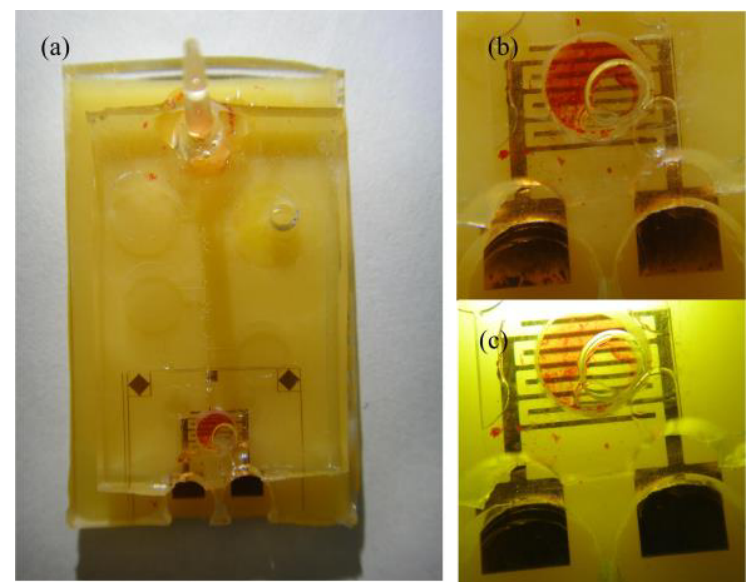

Figure 5-4. Agglutination (B cell and anti-B).

Moreover, the image in Figure 5-4(b) is similar to those in Figures 5-2(b) and 5-3(b). Under the front light source, small RBC agglutination is not evident. Hence, false conclusion might be determined. Although the photo image is a fundamental method to indicate the blood type, a precise method deserves to be explored.

\subsection{Voltammetry}

Voltammetry is an electroanalytical method used in analytical chemistry. In voltammetry, information about an analyte is obtained by measuring the current as the potential is varied. Based on this principle, RBC agglutination was measured using voltammetry technique.

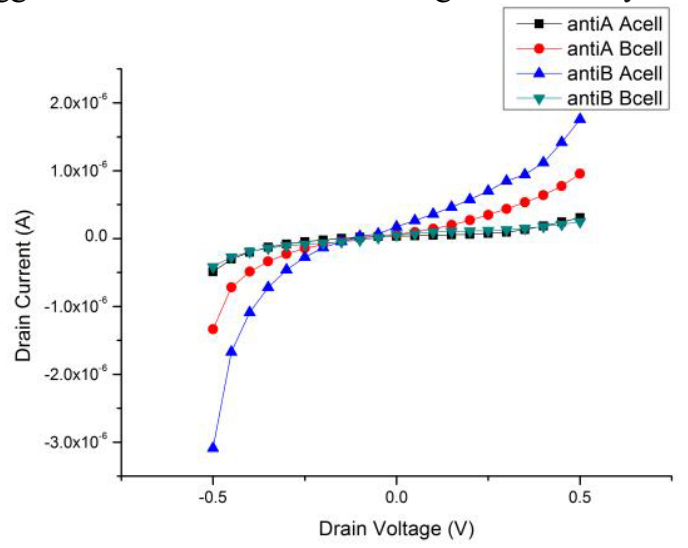

Figure 6. Voltammetry measurement. 
As shown in Figure 6, the curve of RBC agglutination (A cell and anti-A) coincides with that of agglutination of $\mathrm{B}$ cell and anti-B. However, the discrimination between agglutination and non-agglutination is obvious. Although small amount of blood was used in bioassay, accurate result can be obtained.

\subsection{Experimental summary}

To observe a small quantity of RBC agglutination, photo image is not a perfect method. However, voltammetry can provide a precise measurement so that agglutination and non-agglutination can be discriminated.

\section{Conclusions}

This proposed microfluidic blood typing chip has NWIDE for conducting voltammetry measurement of RBC agglutination. Usually, determination of agglutination relies on photo images. False determination might occur if minute amount of blood is used. However, the discrimination between agglutination and nonagglutination was obvious when the voltammetry measurement was employed. This approach provides more accurate result.
1. T.R. Kline, M.K. Runyon, M. Pothiawala, R.F. Ismagilov, Analytical chemistry, 80 (2008)

2. P.P. Goodell, L. Uhl, M. Mohammed, A.A. Powers, Am. J. Clin. Pathol., 134 (2010)

3. W. Malomgré, B. Neumeister, Anal. Bioanal. Chem., 393 (2009)

4. S.G. Sandler, A. Langeberg, N. Avery, P.D. Mintz, Transfusion, 40 (2000)

5. S. Makulska, S. Jakiela, P. Garstecki, Lab Chip, 13 (2013)

6. M. Cuscunà, A. Convertino, E. Zampetti, A. Macagnano, A. Pecora, G. Fortunato, L. Felisari, G. Nicotra, C. Spinella, F. Martelli, Appl. Phys. Lett., 101 (2012)

7. C. V. Tuan, M.A. Tuan, N.V. Hieu, T. Trung, Curr. Appl. Phys., 12 (2012)

8. V.A. Antohe, A. Radu, S. Mátéfi-Tempfli, L. Piraux, IEEE Trans. Nanotechnol., 10 (2011)

9. A.A. Ansari, A. Kaushik, P.R. Solanki, B.D. Malhotra, Bioelectrochemistry, 77 (2010)

10. H.-K. Chang, F.N. Ishikawa, R. Zhang, R. Datar, R.J. Cote, M.E. Thompson, C. Zhou, ACS Nano, 5 (2011)

11. Y.W. Heo, D.P. Norton, L.C. Tien, Y. Kwon, B.S. Kang, F. Ren, S.J. Pearton, J.R. LaRoche, Mater. Sci. Eng. R-Rep., 47 (2004)

12. J. Song, S. Lim, J. Phys. Chem. C, 111 (2007)

\section{References}

